\title{
Applications of Computational and NMR Methodologies to the Study of Homoallylic Alcohols Diastereomers ${ }^{\dagger}$
}

\author{
Viviana Dorn 1,*, Emilio Lorenzo Martínez ${ }^{2}$ and Gabriel Radivoy 1,* \\ 1 Instituto de Química del Sur (INQUISUR-CONICET), Departamento de Química, Universidad Nacional \\ del Sur, Avenida Alem 1253, B8000CPB Bahía Blanca, Argentina \\ 2 Unidad de Resonancia Magnética, Universidad de Alicante (UA), Carretera de San Vicente del Raspeig s/n, \\ 03690 San Vicente del Raspeig, Alicante, España; emilio.lorenzo@ua.es \\ * Correspondence: vdorn@uns.edu.ar (V.D.); gradivoy@criba.edu.ar (G.R.) \\ + Presented at the 22nd International Electronic Conference on Synthetic Organic Chemistry, 15 November- \\ 15 December 2018; Available Online: https://sciforum.net/conference/ecsoc-22.
}

Published: 14 November 2018

\begin{abstract}
Through reducing the system $\mathrm{InCl}_{3}-\mathrm{Li}-\mathrm{DTBB}$ (cat.) in THF at room temperature and in the absence of any additives or anti-caking ligand, we have synthesized indium nanoparticles (InNPs) of about $4 \mathrm{~nm}$. The catalyst was employed in the allylation of carbonyl compounds, giving excellent yields of the corresponding homoallylic alcohols. We have established that the reaction products come from a $\gamma$-coupling via a six members cyclic transition state, type Zimmerman-Traxler. Relative to the selectivity, the allylation with crotyl bromide of ortho substituted benzaldehydes (e.g., $o-\mathrm{NO}_{2}$, $o$-OMe, $o-\mathrm{Cl}_{0} \mathrm{o}-\mathrm{CF}_{3}$ ) showed syn selectivity. With the aim to improve the mentioned selectivity, we synthetized $o$-PrO-benzaldehyde, and evaluated the reaction with crotyl bromide and InNPs. The homoallylic alcohol 1-(2-isopropoxyphenyl)-2-methylbut-3-en-1-ol was obtained almost quantitatively after $1 \mathrm{~h}$ as a mixture of the syn-and anti-isomers. The relationship observed by ${ }^{1} \mathrm{H}-$ RMN was 75:25, but we did not know if the $s y n$-isomer was the dominant because the product has not been reported in the scientific literature. Based on this, and in order to determinate which $1 \mathrm{H}-$ NMR signals correspond to each isomer, we started computational theoretical and NMR studies. The initial conformational analysis was performed using the semiempirical PM3 method, then we work with the B3LYP functional, applying the $6-31+G^{*}$ basis set and the solvent effect (chloroform) was evaluated with the PCM model as implemented in Gaussian09. So, we found thirteen lowenergy conformations for the syn-diastereomer and six low-energy conformations for the antidiastereomer. On the other hand, we have carried out NMR experiments such as ${ }^{1} \mathrm{H},{ }^{13} \mathrm{C}, \mathrm{HSQC}$, to assign the signals of each diastereomer; and experiments such as NOESY, selective NOE, JRes, homo- and hetero-nuclear J-coupled and J-decoupling, to be able to measure coupling constants and establish the structure of each diastereomer.
\end{abstract}

Keywords: indium nanoparticles; DFT; NMR

\section{Introduction}

In the last few years we have been working on the indium-mediated allylation of carbonyl compounds with allyl bromides as an interesting and convenient method to obtain homoallylic alcohols, significant building blocks in organic synthesis. Besides the reactivity, the diastereoselectivity in the addition of an allyl-metal to a carbonyl compound is a fundamental parameter to consider, and could be explained by steric and stereoelectronic or chelating effects [1]. In this sense, previously we report [2] a series of reactions of carbonyl compounds and allylic 
bromides mediated by indium nanoparticles (InNPs) of $4.0 \pm 0.5 \mathrm{~nm}$. The reagents were selected rationally in order to complete an appropriate mechanistic knowledge of the system. Substituted allyl bromides allowed us to establish that the reaction products come from a $\gamma$-coupling, via a sixmembered cyclic transition state, type Zimmerman-Traxler. On another hand, in order to assess the syn-anti selectivity of the InNPs-mediated allylation of carbonyl compounds, we investigated the reaction of a series of ortho-substituted benzaldehydes bearing either electron-donating or electronwithdrawing groups with crotyl bromide. The reaction led to the corresponding alcohols in excellent yields in rather short reaction times, giving syn-diastereomers as the major products. In this sense, ortho-propoxybenzaldehyde yielded the expected product with a 75:25 diastereoselectivity determined by ${ }^{1} \mathrm{H}-\mathrm{RMN}$. Considering that the homoallylic alcohol 1-(2-isopropoxyphenyl)-2methylbut-3-en-1-ol was not reported in the scientific literature, we apply different DFT and NMR methodologies that could allow us to assign each diastereomer, the discussion about these topics is included below.

\section{Methods}

\subsection{General}

All moisture sensitive reactions were carried out under a nitrogen atmosphere. Anhydrous tetrahydrofuran was freshly distilled from sodium/benzophenone ketyl. All starting materials were of the best available grade (Aldrich, Merck) and were used without further purification. Column chromatography was performed with Merck silica gel $60(0.040-0.063 \mathrm{~mm}, 240-400 \mathrm{mesh})$. Thin layer chromatography (TLC) was performed on precoated silica gel plates (Merck 60, F254, $0.25 \mathrm{~mm}$ ).

\subsubsection{Instrumentation and Analysis}

Nuclear magnetic resonance (NMR) spectra were recorded on a Bruker ARX-300 spectrophotometer using $\mathrm{CDCl}_{3}$ as solvent and tetramethylsilane (TMS) as internal reference. Mass spectra (EI) were obtained at $70 \mathrm{eV}$ on a Hewlett Packard HP-5890 GC/MS instrument equipped with a HP-5972 selective mass detector. The purity of volatile compounds and the chromatographic analyses (GC) were determined with a GC Shimadzu (GC-14B) with a flame ionization detector equipped with a HP-5MS column $(30 \mathrm{~m} \times 0.25 \mathrm{~mm} \times 0.25 \mu \mathrm{m})$ using nitrogen as carrier gas.

\subsubsection{Synthesis of $O$-isopropoxybenzaldehyde (1)}

A mixture of $\mathrm{K}_{2} \mathrm{CO}_{3}(2.8 \mathrm{~g}, 20 \mathrm{mmol})$ and salicylaldehyde $(1.22 \mathrm{~g}, 1.4 \mathrm{~mL}, 10 \mathrm{mmol})$ in anhydrous DMF $(10 \mathrm{~mL})$ was stirred at room temperature under nitrogen atmosphere. The reaction mixture changed to yellow. Then isopropyl bromide $(6.4 \mathrm{~mL}, 10 \mathrm{mmol})$ was added drop to drop by syringe, and the suspension was stirred for 2 days. Then, the resulting suspension was diluted with dichloromethane $(30 \mathrm{~mL})$ and treated with $\mathrm{NaOH} 2 \mathrm{M}$ and water. The combined extracts were dried over anhydrous $\mathrm{Na}_{2} \mathrm{SO}_{4}$, and evaporated (20 mbar). The resulting residue was purified by flash column chromatography (silica gel, hexane-ethyl acetate) to give the corresponding aldehyde (1.6 g, $98 \%)$.

\subsubsection{Synthesis of 1-(2-Isopropoxyphenyl)-2-methylbut-3-en-1-ol (2)}

A mixture of lithium powder $(24.5 \mathrm{mg}, 3.5 \mathrm{mmol}), \mathrm{DTBB}(26.7 \mathrm{mg}, 0.1 \mathrm{mmol})$ and $\mathrm{InCl}_{3}(221 \mathrm{mg}$, $1.0 \mathrm{mmol})$ in THF (2 mL) was stirred at room temperature under nitrogen atmosphere. The reaction mixture, which was initially dark green, changed to black, indicating the formation of the indium nanoparticles (InNPs). Then crotyl bromide $(202.5 \mathrm{mg}, 0.150 \mu \mathrm{L}, 1.5 \mathrm{mmol})$ in THF $(2 \mathrm{~mL})$ was added by syringe, and the suspension was stirred for $30 \mathrm{~min}$. Subsequently, $o$-isopropoxybenzaldehyde ( 54 $\mathrm{mg}, 0.33 \mathrm{mmol})$ in THF $(2 \mathrm{~mL})$ was added by syringe. After total conversion of the starting material (TLC, GC), the resulting suspension was diluted with diethyl ether $(10 \mathrm{~mL})$ and treated with $10 \% \mathrm{HCl}$ and water. The combined extracts were dried over anhydrous $\mathrm{Na}_{2} \mathrm{SO}_{4}$, and evaporated (20 mbar). 
The resulting residue was purified by flash column chromatography (silica gel, hexane-ethyl acetate) to give the corresponding homoallylic alcohol (95\%).

\subsubsection{Computational Procedure}

The calculations were performed with the Gaussian09 program [3] The initial conformational analysis of selected compounds was performed with the semiempirical PM6 method. The geometry of the most stable conformer thus obtained was used as starting point for the B3LYP studies. Zeropoint energy were computed at the $6-31+G^{*}$ level for all atoms and LanL2DZ for indium. The characterization of all stationary points was done by Hessian matrix calculations of geometries obtained with full optimization for the minimums and by using the QST2 or QST3 methodologies for the transition state. The energies in solution were obtained within the Tomasi's polarized continuum model (PCM) as implemented in Gaussian09. The figures were built with the VMD program.

\section{Results and Discussions}

The reaction between ortho-propoxybenzaldehyde (1) and crotyl bromide catalyzed by the InNPs, in THF as solvent and then $1 \mathrm{~h}$ of reaction time, led to the corresponding homoallylic alcohol (2) in excellent yields with a 75:25 diastereoselectivity determined by ${ }^{1} \mathrm{H}-\mathrm{RMN}$ (Figure 1).<smiles>CC(C)Oc1ccccc1C=O</smiles>
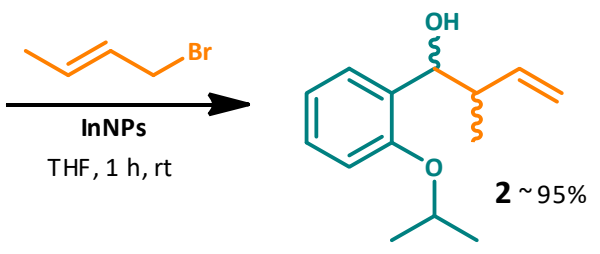

syn:anti $75: 25$

Figure 1. Representative crotylation.

InNPs mediated allylation of ortho-isopropoxybenzaldehyde with crotyl bromide.

As we have been doing, we performed a computational analysis using DFT [4] methods with the Gaussian09 program. The initial conformational analysis was performed using the semiempirical PM3 method, and we then worked with the B3LYP [5-7] functional, applying the LanL2DZ pseudopotential for the indium and the $6-31+G^{*}$ basis set for all the other atoms. The solvent effect was evaluated with the PCM model. To simplify the reactive system and considering results reported by other authors, we evaluated the potential energy surfaces (PES) for the mentioned process by considering the formation of an initial complex between the carbonyl compound and the allyl-indium intermediate, a six-member cyclic chair-like transition state (TS) and a final complex, as can be seen in Scheme 1 for a representative compound. An auxiliary bromide atom was used as ligand for the indium atom to obtain a simplified neutral model system [8,9].<smiles>C=C/C=C/c1ccccc1</smiles>

Initial complex

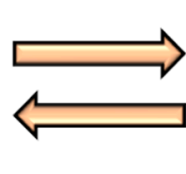

Zimmermann-Traxler type TS

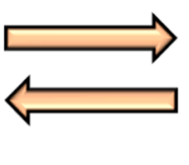

Scheme 1. Representative structures applied in the computational analysis.

As we mentioned previously, for the crotylation of o-propoxybenzaldehyde, a mixture of diastereomer alcohols (2) was obtained, with a higher proportion of syn regarding to anti (75:25). The computational analysis agreed with these results, being the Eas 9.0 and $12.5 \mathrm{kcal} / \mathrm{mol}$ respectively. While both processes were endothermic, the first process was much less endothermic $(+0.9 \mathrm{kcal} / \mathrm{mol})$ than the second $(+2.4 \mathrm{kcal} / \mathrm{mol}$ ) (Figure 2). Then, we carried out a more specific conformational 
inspection of the diastereomers of the product, the homoallylic alcohol 2. At first, we obtained a lot of initial conformers by changing three dihedral angles 1, 2 and 3 (Figure 3) at the same time, and we optimized them using the semiempirical PM3 method. Then we worked with the B3LYP functional, applying the $6-31+G^{*}$ basis set and we found thirteen low-energy conformations for the syndiastereomer and six low-energy conformations for the anti-diastereomer, of which five syndiastereomers (Figure 4) and two anti-diastereomers (Figure 5), would structurally match with the data that we obtained from the different NOE's experiments.

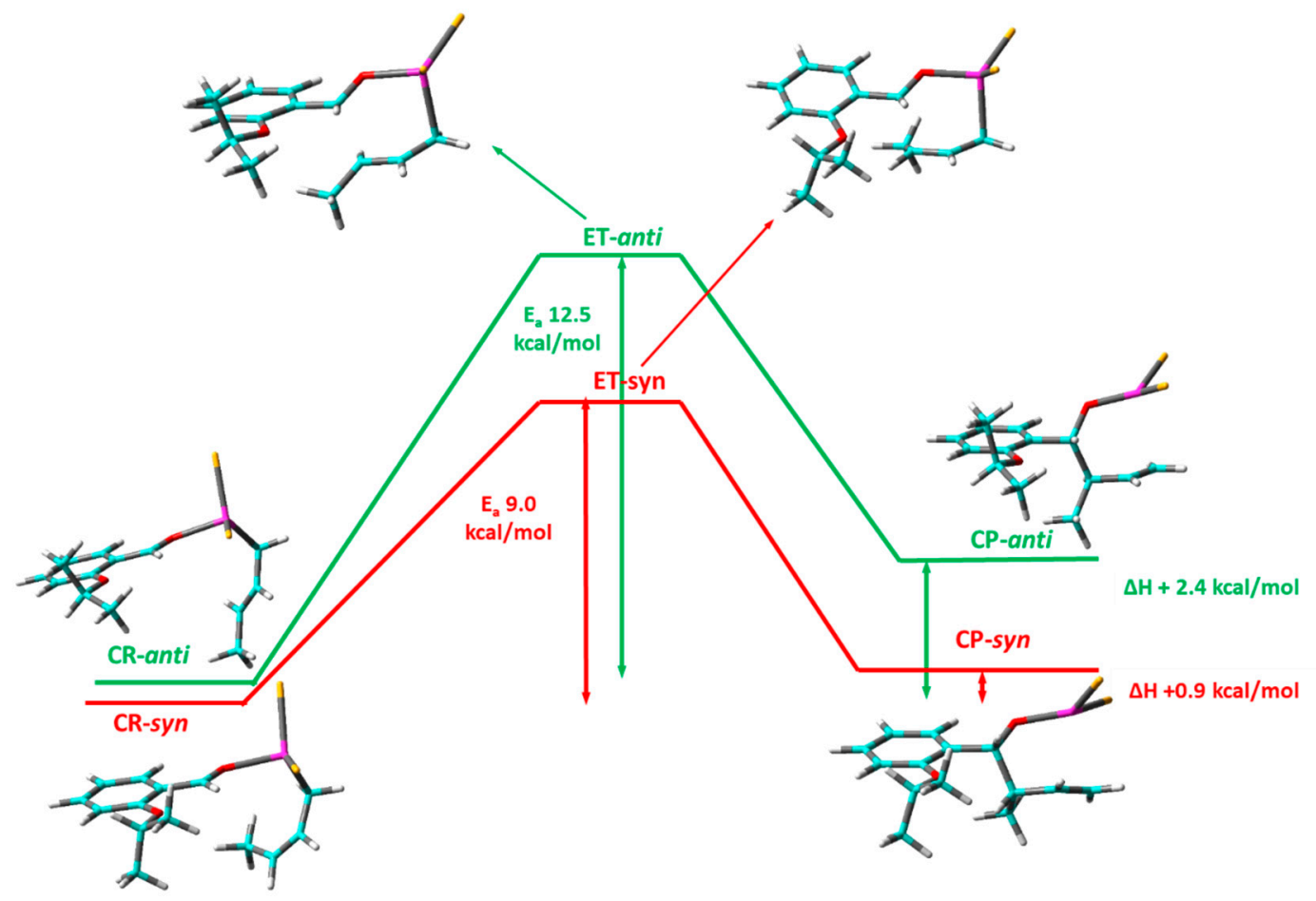

Figure 2. B3LYP/6-31+G*LanL2DZ(In)/PCM(THF) potential energy profiles $(\mathrm{kcal} / \mathrm{mol})$ for the formation of CP-syn (red line) and CP-anti (green line).

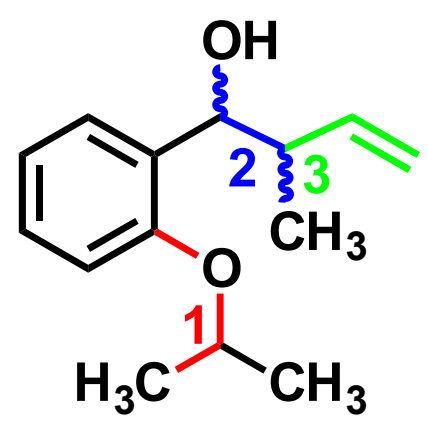

Figure 3. Homoallylic alcohol 2. 

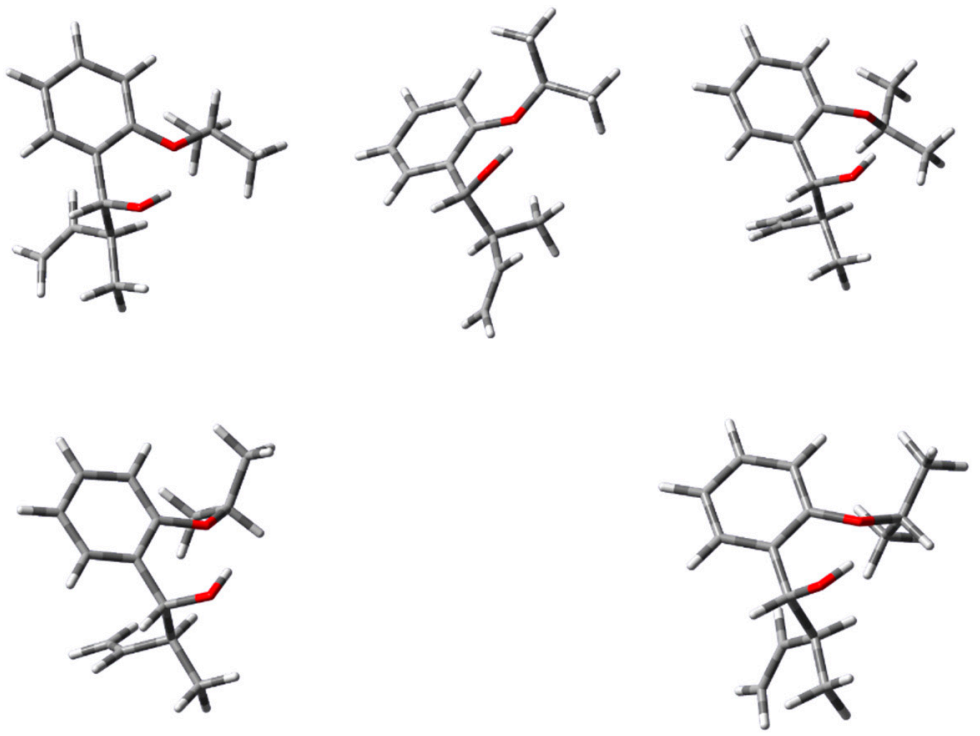

Figure 4. The five low-energy conformations for the syn-diastereomer of 2.
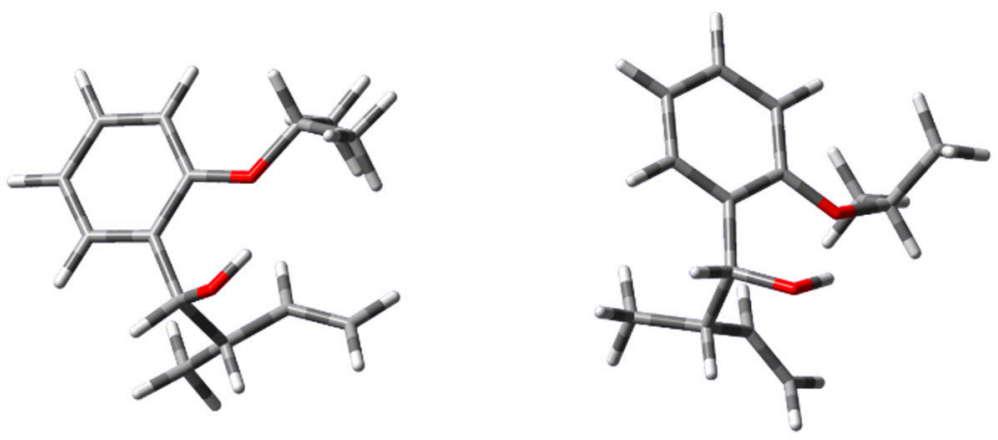

Figure 5. The two low-energy conformations for the anti-diastereomer of 2.

With regard to the NMR analysis, from the signal $\mathbf{A}$ of the ${ }^{1} \mathrm{H}-\mathrm{NMR}$ of the mixture of diastereomers of 2 , we obtained the syn:anti relationship 75:25 (Figure 6). 


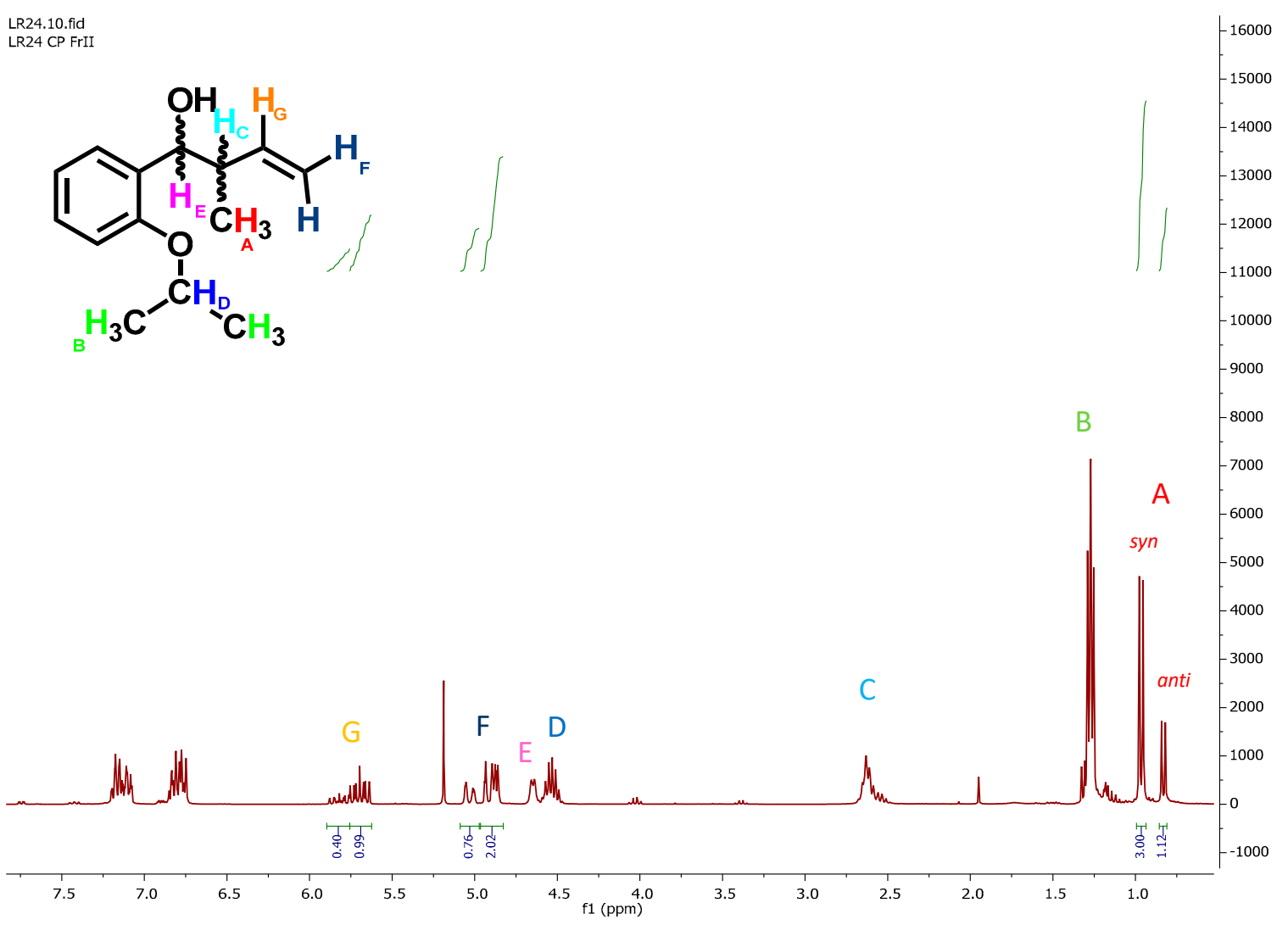

Figure $6{ }^{1} \mathrm{H}-\mathrm{NMR}$ of diastereomers mixture of 2.

It is known that the coupling constant $(J)$ values are extremely useful for the conformational analysis of organic compounds, since the homonuclear scalar couplings ${ }^{3} \mathrm{JHH}_{\mathrm{HH}}$ values are directly related to the dihedral angles through the Karplus equation. Considering the J-based methodology reported by Riccio et al. [10] applied to the determination of the relative configuration of two vicinal methines, we found intermediate values of ${ }^{3} \mathrm{~J}(\mathrm{E}) \mathrm{H}(\mathrm{C}): 6.7 \mathrm{~Hz}$ (syn-diastereomer) and $7.3 \mathrm{~Hz}$ (antidiastereomer), these values are related to two possible orientations gauche or anti for $\mathrm{HE}$ and $\mathrm{Hc}$ respectively. Likewise, the heteronuclear scalar couplings ${ }^{2} \mathrm{~J}_{\mathrm{CH}}$ values are particularly useful when the carbon bears an electronegative substituent like an oxygen; in this case, this ${ }^{2} \mathrm{~J}_{\mathrm{CH}}$ value, can be related to the dihedral angle between the proton $(\mathrm{Hc})$ and the oxygen $(\mathrm{OH})$ bound to the carbon. We found large values of ${ }^{2} \mathrm{~J}(\mathrm{E}) \mathrm{H}(\mathrm{C}): 128.7 \mathrm{~Hz}$ (syn-diastereomer) and $124.5 \mathrm{~Hz}$ (anti-diastereomer), these values are related to a gauche orientation for $\mathrm{He}$ and $\mathrm{OH}$ respectively. That observations are in agreement to the structures found through the computational calculations (Figures 3 and 4).

Regarding to the nuclear Overhauser effect (NOE), from a series of selective NOE experiment, we could observe a series of NOE effect for the syn-diastereomer between $\mathrm{HE}_{\mathrm{E}} \mathrm{HG}_{\mathrm{G}}$; $\mathrm{HC}_{\mathrm{C}}-\mathrm{H}_{\mathrm{F}}$; $\mathrm{H}_{\mathrm{A}}-\mathrm{HE}_{\mathrm{E}}$; $\mathrm{HC}_{\mathrm{C}}$ $\mathrm{H}_{\mathrm{A}}$ and for the anti-diastereomer between $\mathrm{H}_{\mathrm{A}}-\mathrm{H}_{\mathrm{D}} ; \mathrm{H}_{\mathrm{C}}-\mathrm{H}_{\mathrm{A}}$. In the case of the syn isomer, these observations would be in agreement with two of the syn-conformers of Figure 3.

\section{Conclusions}

The in situ prepared InNPs have been demonstrated to be efficient for the synthesis of novel homoallylic alcohols. Both the DFT and NMR methodologies applied allowed us to study the synanti selectivity of the reaction, and have been shown to be a successful approach for assignment of the diastereomers. We are currently performing computational NMR calculations of chemical shift using the GIAO method, in order to ensure the assignment of diastereomers.

Acknowledgments: We are grateful for support provided by the ANPCYT, CONICET and SGCyT-UNS. 


\section{References}

1. Dorn, V.; Chopa, A.; Radivoy, G. Mild bottom-up synthesis of indium(0) nanoparticles: characterization and application in the allylation of carbonyl compounds. RSC Adv. 2016, 6, 23798-23803.

2. Fortunato, L.; Rossi, L.; Radivoy, G.; Dorn, V. Reactivity and selectivity of the InNPs mediated allylation of carbonyl compounds: A DFT study. In Proceedings of the 20th International Electronic Conference on Synthetic Organic Chemistry, online, 1-31 November 2016; Volume 20, p. e007, doi:10.3390/ecsoc-20-e007.

3. Gaussian 09, Revision C.01, M. J. Frisch, G. W. Trucks, H. B. Schlegel, G. E. Scuseria, M. A. Robb, J. R. Cheeseman, G. Scalmani, V. Barone, B. Mennucci, G. A. Petersson; et al. Gaussian, Inc.: Wallingford, CT, USA, 2010.

4. Kohn, W.; Sham, I.J. Self-Consistent Equations Including Exchange and Correlation Effects. Phys. Rev. 1965, 140, A1133-A1138.

5. Lee, C.; Yang, W.; Parr, R.G. Development of the Colle-Salvetti correlation-energy formula into a functional of the electron density. Phys. Rev. B 1988, 37, 785-789.

6. Becke, A.D. Density-functional exchange-energy approximation with correct asymptotic behavior. Phys. Rev. A 1988, 38, 3098-3100.

7. Miehlich, E.; Savin, A.; Stoll, H.; Preuss, H. Results obtained with the correlation energy density functionals of becke and Lee, Yang and Parr. Chem. Phys. Lett. 1989, 157, 200-206.

8. Bowyer, W.J.; Singaram, B.; Sessler, A.M. Nature of the intermediates formed during indium mediated allylation under Barbier conditions. Spectroscopic and experimental data on allylindium species. Tetrahedron 2011, 67, 7449-7460.

9. Dam, J.H.; Fristrup, P.; Madsen, R.J. Combined Experimental and Theoretical Mechanistic Investigation of the Barbier Allylation in Aqueous Media. Org. Chem. 2008, 73, 3228-3235.

10. Riccio, R.; Bifulco, G.; Cimino, P.; Bassarello, C.; Gomez-Paloma, L. Stereochemical analysis of natural products. Approaches relying on the combination of NMR spectroscopy and computational methods. Pure Appl. Chem. 2003, 75, 295-308.

(C) 2019 by the authors. Licensee MDPI, Basel, Switzerland. This article is an open access article distributed under the terms and conditions of the Creative Commons Attribution (CC BY) license (http://creativecommons.org/licenses/by/4.0/). 